\title{
Dressler's pericarditis and an unusual vascular lesion of the myocardium
}

\author{
George Abraham ${ }^{1 *}$ and Yousef Daryani ${ }^{2}$ \\ ${ }^{1}$ Department of Cardiology SpR, Royal Free Hospital, Hampstead, UK \\ ${ }^{2}$ Epsom General Hospital, Dorking Road, Epsom, UK
}

\section{Description of clinical presentation}

An 80-year-old gentleman presented initially to a hospital in Scotland with a week long history of chest pain and shortness of breath. He proceeded to a coronary angiogram which showed severe mid vessel left anterior descending artery (LAD) stenosis, chronic total occlusion (CTO) of the left circumflex distal to the first oblique marginal branch (OM1), and mild disease in the right coronary artery (RCA). He underwent percutaneous intervention to the LAD. He was subsequently admitted to our Hospital in South London three weeks later with pleuritic chest pain, shortness of breath, productive cough and pyrexia.

His ECG showed sinus rhythm with right bundle branch block (RBBB) and no dynamic ischemic changes (Figure 1). There was a mild troponin T rise of $85 \mathrm{ng} / \mathrm{L}$ which fell to $80 \mathrm{ng} / \mathrm{L}$ on a subsequent test roughly 12 hours later. His other biochemistry results included a C-reactive protein level of $130 \mathrm{mg} / \mathrm{L}$ and a WBC count of $12 \mathrm{X}$ $10^{9} / \mathrm{L}$ with a neutrophilia. Clinical examination and chest radiograph demonstrated features consistent with a left basal pneumonia. His echocardiogram showed mildly impaired left ventricular systolic function with a hypokinetic lateral wall and a moderate pericardial perfusion (Figure 2).

Based on these findings and the natural history of the patient's symptoms, a working diagnosis of Dressler's pericarditis was made. In order to further characterise the nature of the pericardial disease as well as the myocardial viability, given the recent coronary event, the patient proceeded to a Cardiac MRI scan.

\section{Cardiac MRI findings}

Cardiac MRI showed mildly increased left ventricular (LV) volume indices (End Diastolic Volume - 148mls, End Diastolic Volume Indexed - $71 \mathrm{mls}$, and End Systolic Volume - $75 \mathrm{mls}$, End Systolic Volume Indexed - 31mls) and mildly impaired LV systolic function with LV ejection fraction $48 \%$, normal right ventricular systolic function, and a well circumscribed lesion in the basal lateral wall (Figures 3 and 4). The lesion was hypointense in TruFISP, hyperintense in T1 and T2 weighted images which suggested the lesion did not contain water or fat. The lesion showed strong homogenous high intake enhancement with gadolinium contrast both in the early and late phase suggesting it was highly vascular. The perfusion study demonstrated a fixed perfusion defect around the lesion. In the gadolinium study, there was no evidence of LV or RV thrombus in the early phase while in the late phase, there was a patch of full thickness late gadolinium enhancement (LGE) around the lesion (Figure 5).

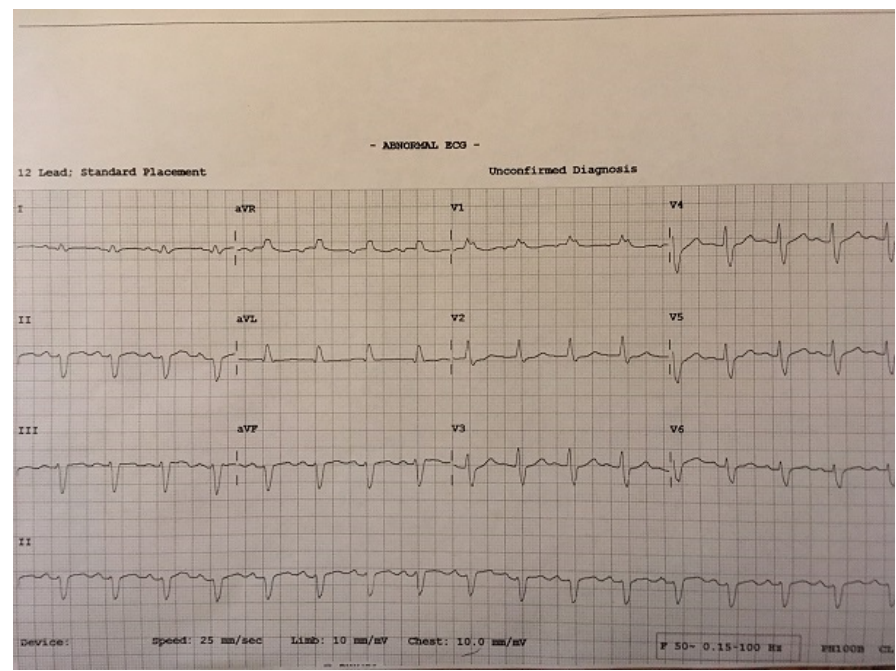

Figure 1. ECG at admission showing sinus rhythm with RBBB.

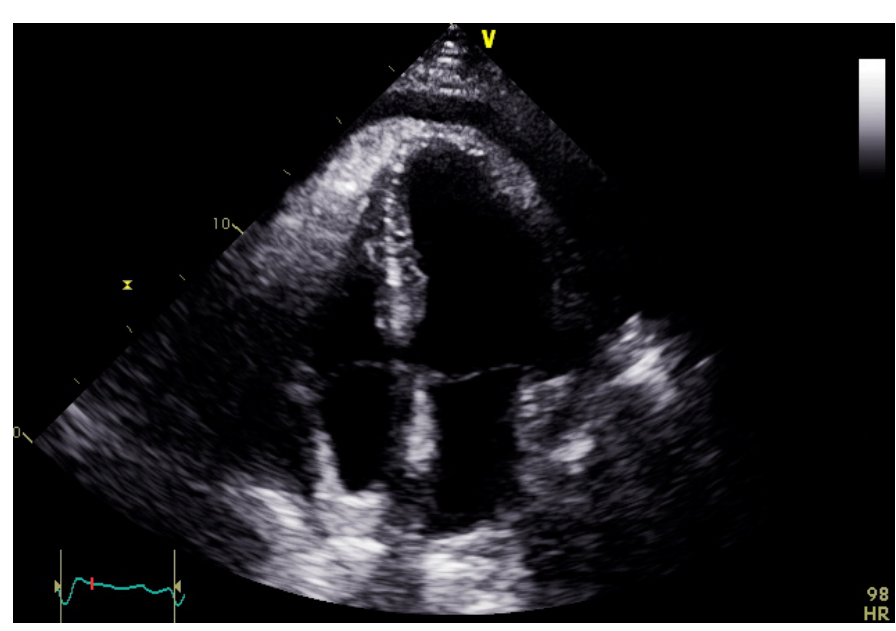

Figure 2. Apical 4 chamber echocardiogram still image demonstrating moderate pericardial effusion.

${ }^{\star}$ Correspondence to: George Abraham, Department of Cardiology SpR, Royal Free Hospital, Hampstead, NW3 2QG, UK, E-mail: george.abraham@nhs.net

Key words: Dressler's pericarditis, cardiac tumours, left ventricular aneurysm, post cardiac injury syndrome, diagnostic utility of CMR

Received: June 10, 2019; Accepted: June 25, 2019; Published: June 28, 2019 


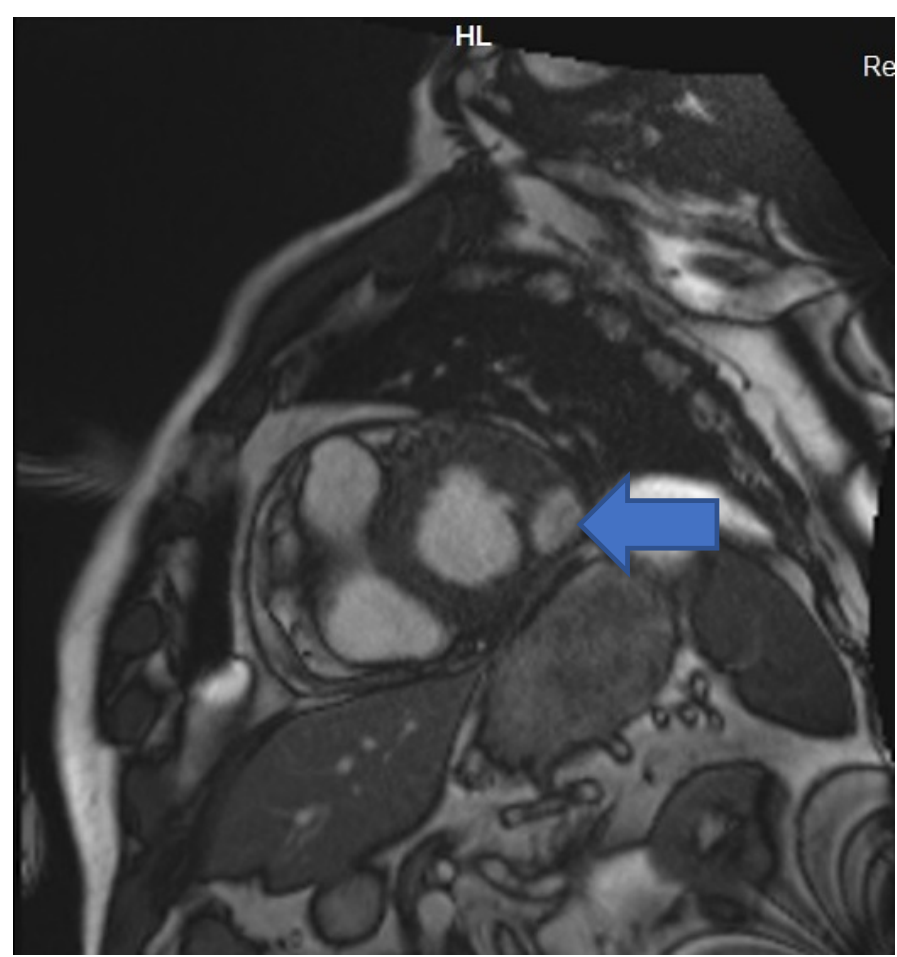

Figure 3. Well circumscribed hypointense lesion in basal lateral wall of left ventricle seen in short axis SSFP cine (Blue arrow).

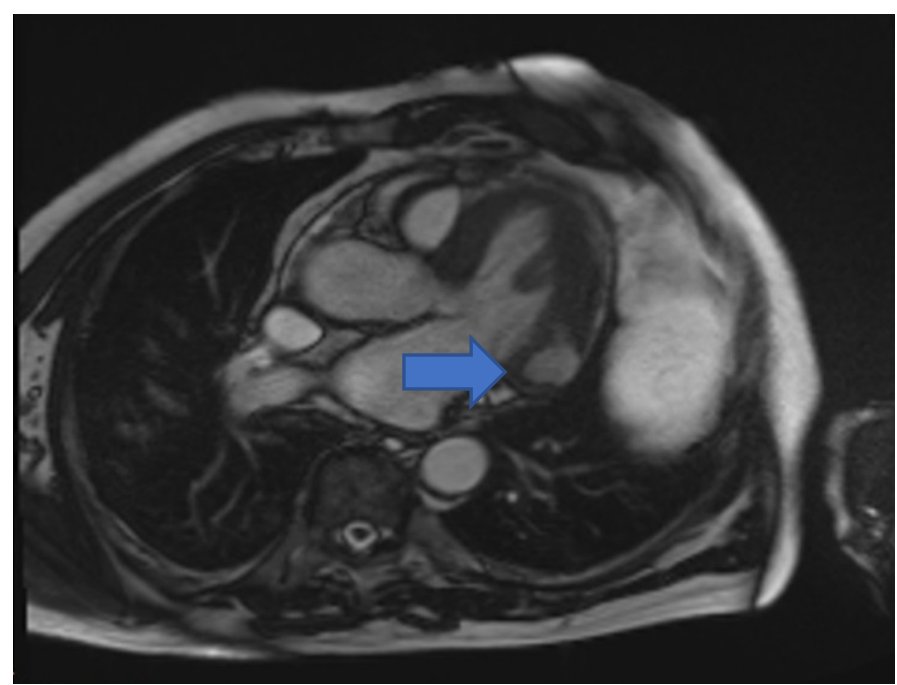

Figure 4. Hypointense lesion seen in basal lateral wall of left ventricle in cross cut long axis view SSFP cine (Blue arrow).

\section{Patient outcome}

Our initial impression of the lesion in the basal lateral wall was of cardiac malignancy in view of its highly vascular appearance and the associated pericardial effusion. A CT scan of thorax, abdomen and pelvis showed no sinister pathology though a hypointense lesion in the basal lateral LV wall was seen again. CT PET scanning revealed no high uptake lesions suggestive of malignancy and the patient remained well with no further clinical symptoms. After review of the coronary angiogram and the initial clinical history of a late presentation infarct, the findings were felt to be more consistent with a full thickness infarct in the basal lateral wall and the lesion in this region more likely represented a left ventricular aneurysm although the neck of the aneurysm was barely visible.

The patient was treated with antibiotics for bronchopneumonia, a course of non-steroidal anti-inflammatory drugs and Colchicine for Dressler's and continued with post MI medical therapy. He remained asymptomatic at 3 months and 6 months follow up.

A CMR 6 months later shows resolution of the lesion but persisting LGE in the left circumflex territory (Figures 4 and 5).

Given the transience of the left ventricular abnormality and its location, it was concluded that the culprit coronary lesion in the first instance was likely to have been the left circumflex: the CMR abnormalities represent a left ventricular aneurysm and a pericardial effusion related to post cardiac injury (Dressler's) syndrome (Figures 6 and 7).

\section{Perspective}

The case demonstrates an unusual appearance of left ventricular aneurysm seen in an unusual basal lateral position, felt initially to be a malignant vascular lesion of the left ventricle. The differential diagnosis

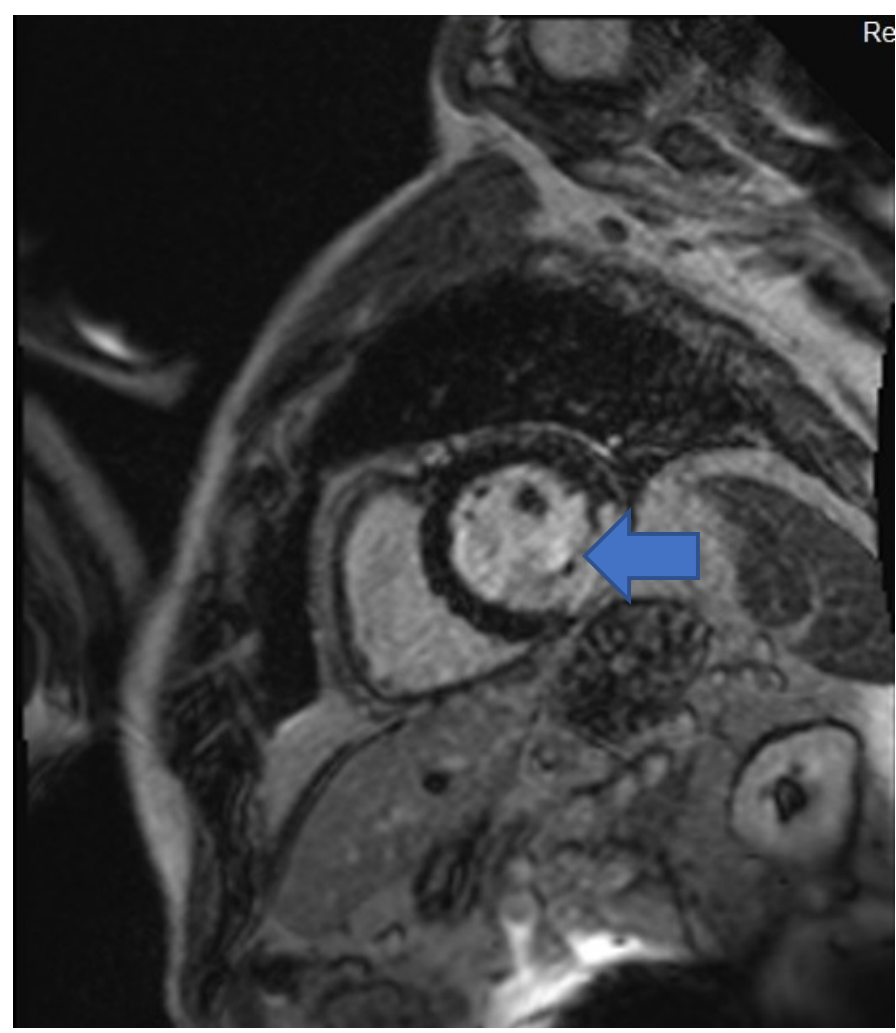

Figure 5. LGE contrast imaging shows full thickness LGE in the basal lateral/inferolateral wall in the region of the lesion. (Blue arrow).

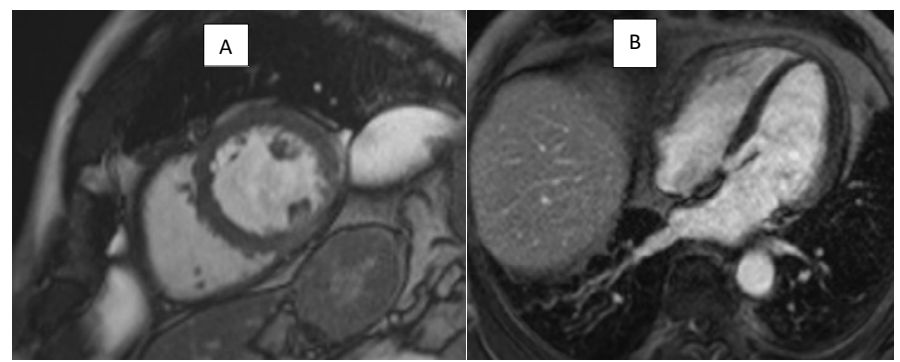

Figures 6. Still images from 6 monthly follow up scan from SSFP cine sequences showing resolution of left ventricular aneurysm. 


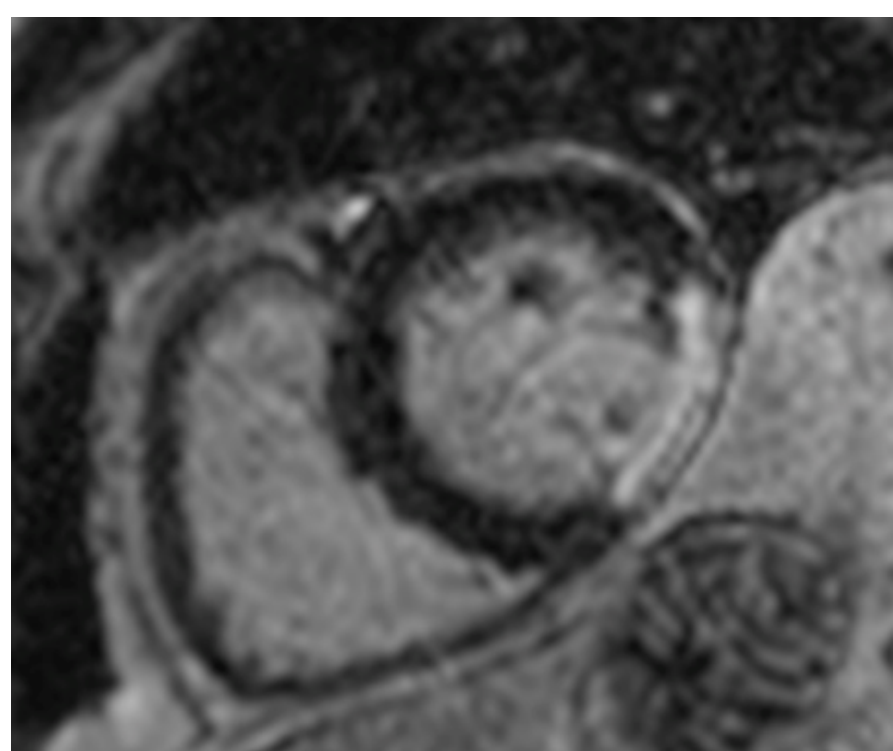

Figure 7. LGE contrast imaging showing fixed perfusion defect in basal lateral wall in follow up study 6 months later.

of a contrast enhancing myocardial mass would include a cardiac metastasis from another primary cancer, myxoma, haemangioma, fibroma, angiosarcoma and sarcoma.

The estimated prevalence of primary cardiac tumours is $1: 2000$, while the incidence of secondary cardiac tumours is up to 20 times higher. It is estimated $2.3-18.3 \%$ of patients with disseminated malignancy have cardiac metastases [1]. Of the primary tumours, $10 \%$ are malignant with the remaining benign lesions however even benign tumours may cause significant pathological consequences, for example: valvular compromise, arrhythmia and pericardial effusion. CMR can be used to characterise the tissue properties of any cardiac mass suggesting a final histological diagnosis.

The most common benign cardiac tumours are atrial myxomas and papillary fibroelastoma. Myxomas appear isointense or hypointense on T1 weighted images, hyperintense on T2 and they tend to heterogeneously enhance with contrast. Papillary fibroelastomas tend to be very small macroscopically and further tissue characterisation is rarely possible. Hemangiomas are more rare, have a variable location and have been reported in intramural, epicardial and pericardial sites. Typical features on CMR are a non-infiltrative mass hyperintense on T2 weighted imaging with contrast enhancement typical of vascular lesions in the Gadolinium study [2]. As such, hemangioma was an important differential diagnosis for the lesion in our case and a definitive answer was only provided by its resolution on follow up imaging.

The most common primary malignant cardiac tumours are undifferentiated pleomorphic sarcoma and angiosarcoma. Sarcomas are usually isointense on T1 weighted imaging, hyperintense on T2 weighted imaging and demonstrate heterogeneous contrast enhancement. Angiosarcomas are heterogeneous in T1 and T2 weighted imaging and show heterogeneous contrast enhancement [3].

True left ventricular (LV) aneurysms represent an outpouching of infarcted myocardium related to an area of abnormal systolic wall motion. Typically, there is an appearance of an aneurysmal sac with a narrow neck, all of which is lined by continuous myocardium as opposed to a false aneurysm which represents the pericardial containment of ventricular free wall rupture. LV aneurysms may be readily identified on invasive ventriculography although they are more commonly diagnosed on transthoracic echocardiography in current clinical practice. The main pathological consequences of LV aneurysms are the risk of arrhythmia and thromboembolism [4]. It was previously estimated that LV aneurysm develops in up to 30 to 35 percent of patients with Q wave MI. However, the incidence of this complication is decreasing in the modern era, and currently is estimated at around 8 to 15 percent in such patients [5].

Expansion of the infarct in the first few hours and days following transmural myocardial infarction is the major pathological predictor of aneurysmal formation. In a prospective echocardiographic study evaluating the time course of aneurysm development following myocardial infarction, no new aneurysm formation was observed later than 3 months after the acute event and the majority of patients with acute anterior MI who developed LV aneurysms had done so by the time of hospital discharge [6].

Approximately 70 to 85 percent of LV aneurysms are located in the anterior or apical walls and are related to total occlusion of the left anterior descending coronary artery and the absence of collateralization. Only 10 to 15 percent involve the inferior-basal walls due to right coronary artery occlusion whereas a lateral LV aneurysm, such as in our case secondary to left circumflex occlusion, is extremely rare [7].

Dressler's pericarditis is the eponymous syndrome of acute pericarditis with or without pericardial effusion first described after MI by Dressler in 1956 [8]. By convention, the term now encompasses pericarditis typically occurring over 1-12 weeks after myocardial injury either from MI, post pericardiotomy in the setting of cardiothoracic surgery or pericardial injury from chest wall trauma.

The co-existence of Dressler's pericarditis and LV aneurysm two distinct pathological sequelae of myocardial infarction, has been reported in the literature on several occasions although the incidence of both complications are decreasing in the reperfusion era $[9,10]$. Studies prior to the use of reperfusion therapy estimated the incidence of Dressler's pericarditis at around 3\% post MI however in one cohort of 201 patients who underwent thrombolysis for acute MI, only one patient developed the syndrome [11]. The historic use of high dose corticosteroid therapy for Dressler's pericarditis is associated with a later predisposition to LV aneurysmal formation and histopathological studies of such cases were the forebear to current guidelines opposing the use of corticosteroids post MI.

Previous case reports as well as our experience with this patient highlight how both complications, although individually rare and in combination, even rarer, can cause significant symptoms. Clinicians should be alert to the diagnosis especially in the assessments of patients re-presenting in the weeks following an MI without revascularisation of the culprit lesion.

\section{References}

1. Basso C, Rizzo S, Valente M (2016) Cardiac masses and tumours. Heart 102: 12301245. [Crossref]

2. Motwani M, Kidambi A, Herzog B, Uddin A, Greenwood J, et al. (2013) MR imaging of cardiac tumours and masses. A review of methods and clinical applications. Radiology 268: 26-43. [Crossref]

3. Esposito A, De Cobelli F, Ironi G, Marra P, Canu T, et al. (2014) CMR in assessment of Cardiac Masses. JACC: Cardiovascular Imaging 7: 733-736.

4. Heatlie G, Mohiaddin R (2005) Left ventricular aneurysm: comprehensive assessment of morphology, structure and thrombus using cardiovascular magnetic resonance. Clinical Radiology 60: 687-692. [Crossref] 
5. Cohn L (1997) Cardiac surgery in the adult. New York [u.a.]: McGraw-Hill, pp: 677.

6. Friedman B, Dunn M (1995) Post Infarction Ventricular Aneurysms. Clinical Cardiology 18: 505-511. [Crossref]

7. Dubnow M, Burchell H, Titus J (1965) Post-infarction ventricular aneurysm. A clinicomorphologic and electrocardiographic study of 80 cases. Am Heart J 70: 753 760. [Crossref]

8. Dressler W (1956) A post-myocardial infarction syndrome. JAMA 160: 1379.
9. Leong I, Tam W, Chan P, Liu Z (2014) An Extraordinary Case of Silent Extensive Anterior Wall Myocardial Infarction Complicated with Giant Left Ventricular Aneurysm and Dressler Syndrome. World Journal of Cardiovascular Diseases 4: 294-298.

10. Bulkley B, Roberts W (1974) Steroid therapy during acute myocardial infarction. Am J Med 56: 244-250. [Crossref]

11. Shahar A, Hod H, Barabash G, Kaplinsky E, Motro M (1994) Disappearance of a Syndrome: Dressler's Syndrome in the Era of Thrombolysis. Cardiology 85: 255-258. [Crossref]

Copyright: (C2019 Abraham G. This is an open-access article distributed under the terms of the Creative Commons Attribution License, which permits unrestricted use, distribution, and reproduction in any medium, provided the original author and source are credited. 